\title{
New Insights on Stochastic Reachability
}

\author{
Manuela L. Bujorianu and John Lygeros
}

\begin{abstract}
In this paper, we give new characterizations of the stochastic reachability problem for stochastic hybrid systems in the language of different theories that can be employed in studying stochastic processes (Markov processes, potential theory, optimal control). These characterizations are further used to obtain the probabilities involved in the context of stochastic reachability as viscosity solutions of some variational inequalities.
\end{abstract}

Keywords: stochastic reachability, stochastic hybrid systems, Markov processes, reduite, optimal stopping problem.

\section{INTRODUCTION}

Many practical systems such as automobiles, chemical processes, biochemical reactions and autonomous vehicles are best described by dynamics that comprise continuous state evolution within a mode of operation and discrete transitions from one mode to another, either controlled or autonomous. Such systems often interact with their environment in the presence of uncertainty and variability. For these systems, the researchers have introduced the modelling paradigm called stochastic hybrid systems (SHS) [4].

Intuitively, SHS can be described as an interleaving between a finite or countable family of diffusion processes (or, sometimes, only dynamical system) and a jump process. Modelling and analysis of SHS have been proved to be a very difficult task from a mathematical point of view. The stochastic analysis apparatus, employed to study different aspects related to the probabilistic features of SHS, is really complex and rather difficult to manage. Studying SHS, one has to combine tools available for diffusion processes and jump processes, in order to obtain valuable properties of these systems. But, the fact the switching mechanism between the diffusion paths is also random (governed by a Markov chain in most cases) make the things complicated and then the use of the well studied diffusion properties is not straightforward.. The main achievement in the study of SHS is that it was proved that their behaviour can be described by some relatively 'good' Markov processes [8], [4]. Moreover, the hybrid nature of these stochastic systems is illustrated by the expression of their generator (or infinitesimal operator) [8]. This generator is an integrodifferential (or pseudo-differential [22]) operator, which is constituted by two other operators, one corresponding to the diffusion part and one for the jumping part of the system. Our experience in investigation of different problems related to SHS has shown that this generator is the main analysis tool available that can smooth the mathematical complexity

Manuela L. Bujorianu is with Faculty of Computer Science, Unversity of Twente, The Netherlands L.M.Bujorianu@cs.utwente.nl

John Lygeros is with Automatic Control Laboratory, ETH Zurich, Switzerland of the SHS study. Mathematical properties of SHS make difficult the extension of the verification techniques available for deterministic hybrid systems. The reachability problem concept in the framework of stochastic hybrid systems was set up in our paper [5]. The mathematical foundations of this concept have been addressed in [6], [5].

In this paper, we continue the theoretical study of the stochastic reachability problem in order to obtain some valuable characterizations of this concept by means of the stochastic analysis tools available for Markov processes. The main achievement attained in this study is the characterization of the reach set probabilities as solutions of some variational inequalities associated to the process generator. Then some dynamic programming methods can be applied to compute these probabilities.

The road that proceeds to the accomplishment of this target is paved with different other characterizations of the reachability problem via some concepts coming from Markov process theory, potential theory and optimal control. These characterizations represent themselves an important contribution of this paper.

The main vehicle, which makes possible the connections between the above characterizations, is given by martingales. Martingales ${ }^{1}$ are important technical tools used in the study of stochastic processes as diffusion processes, jumpdiffusions, etc. It was the merit of the famous mathematician J. Doob who recognised the intrinsic value of the concept of martingale and made it a versatile tool in probability [13].

\section{Problem Formulation}

First, in this section we briefly present the stochastic reachability problem for stochastic hybrid systems. Then, we stress on the main mathematical perspectives from which one can obtain different characterisations of this problem.

Let us consider $M=\left(\Omega, \mathcal{F}, \mathcal{F}_{t}, x_{t}, P_{x}\right)$ a strong Markov process, as the realization of a stochastic hybrid system (see definitions below). For this strong Markov process we address a verification problem consisting of the following stochastic reachability problem.

Given a measurable set $A$ and a time horizon $T>0$, let us to define [5], [6]:

$$
\begin{aligned}
\operatorname{Reach}_{T}(A) & =\left\{\omega \in \Omega \mid \exists t \in[0, T]: x_{t}(\omega) \in A\right\} \\
\operatorname{Reach}_{\infty}(A) & =\left\{\omega \in \Omega \mid \exists t \geq 0: x_{t}(\omega) \in A\right\} .
\end{aligned}
$$

\footnotetext{
${ }^{1}$ The name "martingale" was introduced by Jean Ville as a synonym of "gambling system", in his book on "collectif" in the Borel collection, in 1939 [24].
} 
These two sets are the sets of trajectories of $M$, which reach the set $A$ (the flow that enters $A$ ) in the interval of time $[0, T]$ or $[0, \infty)$.

The reachability problem consists of determining the probabilities of such sets, which can be expressed as

$$
P\left(T_{A}<T\right) \text { or } P\left(T_{A}<\infty\right)
$$

where $T_{A}$ is the first hitting time of $A$

$$
T_{A}=\inf \left\{t>0 \mid x_{t} \in A\right\}
$$

and $P$ is a probability on the measurable space $(\Omega, \mathcal{F})$ of the elementary events associated to $M$.

Another approach to the reachability problem is to look at the first hitting time mean $E_{x} T_{A}$ of the target set $A$, where $E_{x}$ is the expectation w.r.t. $P_{x}$. When $A$ is an unsafe set, the quantities of interest are the lower bounds on the expected value of this hitting time, since these bounds provide a degree of assurance against catastrophic failure. Dually, the mean of the first exit time from a safe domain provides a measure of its stability. It also measures the rate of transition from the domain it exits.

In this paper, we investigate the properties of the quantities (2) from different perspectives: (i) potential theory, (ii) Markov process theory, (iii) stochastic optimal control.

The stochastic reachability problem can be viewed in connection with three concepts $\left.{ }^{2}: 1\right)$ hitting operators (belonging to the Markov process endowment), 2) reduite of a function (belonging to the potential theory language), 3) optimal stopping problem (for Markov processes) with the reward function given by the indicator function of a subset of the state space.

In order to deal with these different perspectives, one needs to look at the whole picture: The main engine to move back and forth between Markov processes, potential theory and optimal control is provided by (sub, super) martingales. The connections between potential theory and probability theory are now well studied and have more than fifty years of history originating in the work of Doob [13], [12] and Hunt [21]. Doob showed how various classical potential theory concepts correspond to properties of superharmonic functions on Brownian motion paths. Hunt extended the ideas to found what is now called probability potential theory, in which each of a large class of Markov processes corresponds to a potential theory and conversely.

Concretely, it is known that if $\left(B_{t}\right)_{t \geq 0}$ is the Brownian motion in $\mathbb{R}^{d}$ and $u$ is a positive superharmonic function in $\mathbb{R}^{d}$, the $u\left(B_{t}\right)$ is a cadlag ${ }^{3}$ supermartingale in $[0, \infty]$ provided that $E\left[u\left(B_{0}\right)\right]<\infty$. This result was proved in the Doob's 1955 paper on the heat equation. It is a major recourse in Hunt's theory, where $u$ became an "excessive" function and the Brownian motion became a Hunt process. In a general way, Doob's work on (sub, super) harmonic functions with the vital underpinning by Brownian paths, paved the way for Hunt's theory on Markov processes and potentials [21].

\footnotetext{
${ }^{2} \mathrm{~A}$ common reference for all these concepts is [12].

3 i.e., its trajectories are right continuous with left limits.
}

In the last half of the 20th century, Hunt's theory has been developed further for the standard Markov processes and then for right Markov processes ${ }^{4}$ (which might be thought of as the modern generalizations of Hunt processes) by different authors (see, for example, [11]).

On the other hand, martingales represent now a versatile tool to study different problems related to Markov processes (convergence theorems, invariance principles, etc). As well. there is now a common practice to study stochastic optimal control problems via martingale methods.

We will see that the reach set probability (2) can be expressed as the reduite of a specific function. With this characterisation in hands, we make the connection with the optimal stopping problem defined for Markov processes. A classical result [14], says that the solution of a general optimal stopping problem for Markov processes can be characterized by means of the smallest superharmonic function dominating the reward function (i.e., the reduite of the reward function). Since the superharmonic/excessive functions can be characterised by means of supermartingales [15], the above characterization can be related with the martingale methods for the optimal stopping problem summarized in [12].

\section{PRELIMINARIES}

In this section we give the necessary background for stochastic hybrid systems, their semantics, some stochastic analysis tools and some martingale theory results.

\section{A. Stochastic Hybrid Systems}

Different modelling paradigms for SHS have been proposed in literature [20], [2]. Applications of SHS range from air traffic management [23], biology [18], to communication networks [19].

Formally, a stochastic hybrid automaton (SHA) is defined as a tuple $H=(Q, \mathcal{X}, F, R, \lambda)$ has been defined in [8], [7].

The executions of an SHA, $H$, can be described as follows: start with an initial point $x_{0} \in X^{q}$, follow a solution of the SDE associated to $X^{q}$, jump when this trajectory hits the boundary or according with the transition rate $\lambda$ (the jump time is the minimum of the boundary hitting time and the time, which is exponentially distributed with the transition rate $\lambda$ ). Under standard assumptions, for each initial condition $x \in \underset{j \in Q}{\cup} X^{j}$, the possible trajectories starting from $x$, form a stochastic process. Moreover, for all initial conditions $x$, the realizations of an SHA make up a family of Markov processes [10], which can be thought of as a Markov process in a general setting.

Let us consider the stochastic process $M=$ $\left(\Omega, \mathcal{F}, \mathcal{F}_{t}, x_{t}, P_{x}\right)$, which represents the realization (or semantics) of $H$, i.e. all its possible trajectories. Under mild assumptions on the parameters of $H, M$ can be viewed as a family of Markov processes with the state space $(X, \mathcal{B})$, where $X$ is the union of modes and $\mathcal{B}$ is its Borel $\sigma$-algebra. Let $\mathcal{B}^{b}(X)$ be the Banach space of

\footnotetext{
${ }^{4}$ These processes satisfy the two "right hypotheses", HD1 and HD2, defined by P.A. Meyer, see [10], p.77.
} 
bounded positive measurable functions on $X$. The meaning of the elements of $M$ can be found in any source treating continuous-parameter Markov processes [10].

\section{B. Setting the Hypotheses}

For the analysis of stochastic hybrid systems, we need to make use of the different characterizations of Markov processes. We briefly present some functional analysis tools (operators associated to a stochastic process), which have been proved to be very useful in the context of continuous time, continuous space Markov processes. Their presence in this paper is justified by the fact that these operators are not standard in the theory of Markov chains, and the reader familiar only with discrete stochastic processes might have difficulties in understanding the contribution of this paper. Let us consider the Markov process $M=\left(\Omega, \mathcal{F}, \mathcal{F}_{t}, x_{t}, P_{x}\right)$. The mathematical objects like operator semigroup $\mathcal{P}=$ $\left(P_{t}\right)_{t>0}$ and operator resolvent $\mathcal{V}=\left(V_{\alpha}\right)_{\alpha>0}$, strong generator $\mathcal{L}$ or extended generator can be defined in standard way (see also [7]). Traditionally, Markov processes have been described by their generators and the corresponding evolutions and rezolvents.

1) Realization of a stochastic hybrid system as a Borel process: Suppose now $M$ represents the realization of a stochastic hybrid system $H$. We have proven that under standard assumption $M$ is a Borel right process [4]. Moreover, we have proved that the sample paths of $M$ are right continuous with left limit, i.e. are cadlags.

Recall that a nonnegative function $f \in \mathcal{B}^{b}(X)$ is called $\alpha$-excessive $(\alpha \geq 0)$ if $e^{-\alpha t} P_{t} f \leq f$ for all $t \geq 0$ and $e^{-\alpha t} P_{t} f \nearrow f$ as $t \searrow 0$. If $\alpha=0$, a 0 -excessive function is simply called excessive function. The excessive functions can be characterised also using the operator resolvent as follows: $f \in \mathcal{B}^{b}(X)$ is an excessive (or a $\mathcal{V}$-excessive) function if $\alpha V_{\alpha} f \leq f$ for all $\alpha>0$ and $\sup \alpha V_{\alpha} f=f$. Let us denote the cone of excessive functions by $\mathcal{E}_{M}$.

We assume also that $M$ is transient. This means that there exists a strictly positive Borel function $q$ such that $V q$ is bounded. The transience of $M$ means that any process trajectory which will visit a Borel set of the state space it will leave it after a finite time. The transience hypothesis guarantees that the cone $\mathcal{E}_{M}$ is rich enough to be used.

2) Some Martingale Theory: For a right Markov process $\left(x_{t}\right)_{t \geq 0}$ (in our case, the realization of the stochastic hybrid automaton, $H$ ) and an excessive function $u$, it is known that the process $\left(u\left(x_{t}\right)\right)_{t \geq 0}$ is a right-continuous $P_{x^{-}}$ supermartingale, for any $x \in X$ such that $u(x)<\infty$ [11]. Then, for the purpose of this paper, we need to remind the following supermartingale inequalities.

Theorem 1 (Supermartingale inequalities): Let $\left\{Y_{t}\right\}_{t \geq 0}$ be a real valued supermartingale. Let $[a, b]$ be a bounded interval in $\mathbb{R}_{+}$. Then

$$
\begin{gathered}
c P\left\{\omega \mid \sup _{a \leq t \leq b} Y_{t}(\omega) \geq c\right\} \leq E Y_{a}+E Y_{b}^{-} \\
c P\left\{\omega \mid \inf _{a \leq t \leq b} Y_{t}(\omega) \leq-c\right\} \leq E Y_{b}^{-}
\end{gathered}
$$

Our main goal is to study the stochastic process $\left(F\left(x_{t}\right)\right)_{t \geq 0}$ and suitable hypotheses for $F$ such that upper bounds for the probabilities (7) can be easily derived.

hold for all $c>0$.

Connections between martingales and Markov processes are well known in the literature. As a result of the Dynkin formula [10] satisfied by the elements in the domain of the strong generator $\mathcal{L}$, a natural martingale can be associated to a Markov process $M=\left(\Omega, \mathcal{F}, \mathcal{F}_{t}, x_{t}, P_{x}\right)$, as follows.

Proposition 2: [10] For $f \in D(\mathcal{L})$ and for any $x \in \mathbf{S}$, the real-valued process defined by

$$
C_{t}^{f}=f\left(x_{t}\right)-f\left(x_{0}\right)-\int_{0}^{t} \mathcal{L} f\left(x_{s}\right) d s
$$

is a martingale on $\left(\Omega, \mathcal{F}, \mathcal{F}_{t}, P_{x}\right)$.

Often, $D(\mathcal{L})$ is difficult to describe completely. Martingale problem has indicated a sort of converse, and that too with a possibly a subspace much smaller than $D(\mathcal{L})$.

Let $X$ be a complete separable metric space and $\mathcal{B}^{b}(X)$ the Banach space of bounded measurable functions on $X$.

We have studied the martingale problem associated to the generator of a realization of a stochastic hybrid system in our paper [8]. We have proved that for an appropriate domain $D(\mathcal{L})$, the generator of stochastic hybrid system has the following integro-differential form

$$
\mathcal{L} f(x)=\mathcal{L}_{\text {cont }} f(x)+\lambda(x) \int_{\bar{X}}(f(y)-f(x)) R(x, d y)
$$

where $\mathcal{L}_{\text {cont }} f(x)$ has the standard form of the diffusion infinitesimal operator.

\section{Stochastic Reachability USing Martingale THEORY}

In this section, we investigate two main directions, based on martingale theory, in tackling with the stochastic reachability problem.

First, in the context of the reachability problem, we consider the case when the target sets are described as level sets of some known measurable functions. The martingale properties of the image of the given stochastic process through this kind of functions are then considered.

Second, we study the expectations of the hitting times of the target sets. The mean hitting times represents the solution of a Dirichlet problem associated to the infinitesimal operator of the process.

\section{A. Upper bounds of the reach set probabilities}

Target sets as level sets. Suppose that the target set $A$ in the state space is described as a level set for a given function $F: X \rightarrow \mathbb{R}$, i.e. $A=\{x \in X \mid F(x) \geq l\} . F$ can be chosen, for example, to be the Euclidean norm or the distance to the boundary of $E$. The probability of the set of trajectories, which hit $A$ until time horizon $T>0$ can be expressed as

$$
P\left\{\sup _{t \in[0, T]} F\left(x_{t}\right) \geq l\right\} .
$$


$F\left(x_{t}\right)$ represents the best candidate for defining a possible abstraction for $M$, which preserves the reach set probabilities. The main difficulty is that $F\left(x_{t}\right)$ is a Markov process only for special choices of $F$. The problem how to choose $F$ well was studied in [7].

In this section, we propose further to 'approximate' the function $F$ such that the stochastic process $\left(F\left(x_{t}\right)\right)_{t \geq 0}$ becomes a supermartingale. Then, some upper bounds for (7) can be easily derived from the martingale's inequalities that are well studied in the literature.

In fact, our scope is to find a stochastic Lyapunov function $\widetilde{F}$, which is close enough to $F$, such that different properties of the supermartingale $\left(\widetilde{F}\left(x_{t}\right)\right)_{t \geq 0}$ could be exploited.

The main goal of this section is to find upper bounds probabilities that appear in the stochastic reachability problem, using the theory of (sub/super)martingales associated to the realization of a stochastic hybrid system.

Methodology description. Suppose now that $A \in \mathcal{B}(X)$ is a target set described as a level set associated to a positive measurable function $F: X \rightarrow \mathbb{R}_{+}$. If $F$ is an excessive function then the problem to find an upper bound for reach set probability (7) is easily solved by (4) since $\left(F\left(x_{t}\right)\right)$ is a $P_{x}$-supermartingale, for any $x \in X$ such that $F(x)<\infty$.

The function $F$ might have different shapes and in order to allow more freedom in choosing it, we can NOT suppose a prior that $F$ is an excessive function. As well it might be difficult to check its excessiveness using different stochastic analysis tools and it is not worthy to spend too much effort in this direction.

In the following, we show that the function $F$ can be "approximated" with an excessive function.

The suitable approximation of $F$ is then exploited, in a separate subsection, to derive the main results of this section with respect to the reachability problem.

First, we introduce the concept of reduced function (that is a potential theory concept), which allows us to find a suitable "excessive approximation" of $F$ which can be used after to provide a supermartingale associated to the process $\left(x_{t}\right)$. For any $f: X \rightarrow \mathbb{R}_{+}$, we denote by $R f$ the function

$$
R f:=\inf \left\{u \in \mathcal{E}_{M} \mid u \geq f\right\}
$$

called the réduite of $f$ with respect to the resolvent $\mathcal{V}$. The reduite of $f$ differs from $f$ only on a negligible set. In potential theory, in the proof of the existence of the reduite for a function $f$, it is usually assumed that $f$ is the difference of two excessive functions.

For the function $F$, we consider that its reduite with respect to the resolvent $\mathcal{V}$ represents a suitable excessive approximation. In many practical applications, it is expected that the function $F$ has some continuity properties. Then it can be shown that $F$ can be approximated as a limit of a sequence of excessive functions.

Upper bounds. Consider now an SHS, $H$ as in section III$\mathrm{B}$, whose realization is given by the Markov process $M$. Suppose that $A \in \mathcal{B}(X)$ is a target set, for which we want to estimate the reach set probabilities (2). Let $F$ a measurable real valued function on $X$ that is used to describe the target set $A$ as a level set.

We might consider first the case when $F: X \rightarrow \mathbb{R}_{+}$is a positive measurable function which can be written as the difference of two bounded excessive functions $F=u-v$, $v \leq u<\infty$. If the excessive functions $u$ and $v$ are known, to evaluate some bounds for the probability (7), we have to use the fact that $\left(u\left(x_{t}\right)\right)_{t \geq 0}$ and $\left(v\left(x_{t}\right)\right)_{t \geq 0}$ are $P_{x^{-}}$ supermartingale for all $x \in X$ (we have supposed that $u, v$ are bounded) and to apply Theorem 1.

We suppose now that the reduite $R F$ exists and we denote it by $\widetilde{F}$. Since $\widetilde{F}$ is an excessive function, the following result, which gives an upper bound of the reach set probability (7), is just a consequence of the Theorem 1 .

Theorem 3: For any $x \in X$ such that $\widetilde{F}(x)<\infty$, we have

$$
P_{x}\left\{\sup _{t \in[0, T]} \widetilde{F}\left(x_{t}\right) \geq l\right\} \leq \frac{\widetilde{F}(x)+\lim _{t \nearrow T} P_{t} \widetilde{F}(x)^{-}}{l} ; l>0
$$

where $\left(P_{t}\right)$ is the operator semigroup.

In the Theorem 3, we have used the fact that $P_{0}$ is the identity operator.

Moreover, the inequality (9) can be written in terms of the infinitesimal generator $\mathcal{L}$, which is known for SHS, see [8]. This fact is possible because there exists a classical result [17], which says that the semigroup $\left(P_{t}\right)_{t>0}$ formula can be computed using the infinitesimal generator $\mathcal{L}$ expression via the following connection: $P_{t}=\exp (t \mathcal{L}), t>0$.

\section{B. Expectations of the hitting times}

If in the expression (5) of the martingale associated to $M$, one can solve the equation $\mathcal{L} f=-1$ then $\widetilde{C}_{t}^{f}=f\left(x_{t}\right)+t$ is martingale.

Consider $A \in \mathcal{B}(\mathbf{S})$ so that $T_{A}$ is its first hitting time. Then the solution of the inhomogeneous Dirichlet problem: $\mathcal{L} u_{A}(x)=-1, x \in \mathbf{S} \backslash A ; u_{A}(x)=0, x \in A$ is

$$
u_{A}(x)=\left\{\begin{array}{l}
E_{x} T_{A}, x \in \mathbf{S} \backslash A \\
0, x \in A .
\end{array}\right.
$$

provided that $T_{A}$ satisfies the sampling integrability conditions for $\widetilde{C}_{t}^{f}$. The Dirichlet problem can be written in term of exit times from the complementary set of $A$.

\section{STOCHASTIC REACHABILITY AS AN OPTIMAL STOPPING PROBLEM}

Suppose that the target set $A$ is not necessary described as a level set. In this case, we aim to characterize the reach set probabilities (2), in connection with the well studied apparatus available for studying Markov processes, in order to derive computational methods to evaluate these probabilities.

The purpose of this section is to investigate some characterizations of the stochastic reachability problem, employing combined mathematical tools originated from potential theory,on one hand, and from control theory of Markov processes, on the other hand.

We work in the framework described in the section III-B 


\section{A. Balayage and reachability}

In this subsection, we characterize the reach set probabilities (2) using the concept of balayage from potential theory and the concept of hitting operator from Markov processes theory.

The reader not interested in the technical details might read only the proposition in the final of this subsection.

Reduite on a set. For any subset $A$ of $X$ and $v \in \mathcal{E}_{M}$, the function $R_{A} v=R\left(1_{A} v\right)$ is called the réduite of $v$ on $A$, where the operator $R$ is defined by (8). We use the convention $0 \cdot(+\infty)=(+\infty) \cdot 0=0$.

Balayage. It is known that for any $\mathcal{B}(X)$ and $v \in \mathcal{E}_{M}$ the function $R_{A} v$ is $\mathcal{B}(X)$-measurable and it is $\mathcal{V}$-supermedian, i.e. $e^{-\alpha t} P_{t}\left[R_{A} v\right] \leq R_{A} v$ for all $t \geq 0$ and $\alpha>0$ ). In this case, we denote by $B_{A} v$ the $\mathcal{V}$-excessive regularization of $R_{A} v$, i.e. $B_{A} v:=\sup \alpha V_{\alpha} R_{A} v . B_{A} v$ is called also balayage of the excessive function $v$ on $A$. The concept of balayage of excessive function is due to Hunt [21]. He showed that:

Proposition 4: The balayage of an excessive function $v$ on a Borel set $A$ is given by $P_{A} v$, where $P_{A}$ is the hitting operator associated to the underlying Markov process $\left(x_{t}\right)$ : $B_{A} v=P_{A} v=E_{x}\left\{v \circ x_{T_{A}} \mid T_{A}<\infty\right\}$, where $T_{A}$ is the first hitting time of $A$ given by (3).

Moreover, $B_{A} v$ is the lower semicontinuous regularization of $R_{A} v$ with respect to a suitable topology on $X$ (namely, the fine topology, which is the smallest topology that makes all excessive functions continuous).

Properties. Briefly, the relations between $v, R_{A} v, B_{A} v$ are: - On the whole state space $X$, we have : $v \geq R_{A} v \geq B_{A} v$; - On the complementary set of $A$, we have: $R_{A} v=B_{A} v$ on $X \backslash A$;

- Moreover, the equality

$$
v=R_{A} v=B_{A} v
$$

holds also on $A \backslash N$, where $N$ is a negligible set of $A$.

Since for a right Markov process, the function identically equal to 1 is excessive, the following characterization of the stochastic reachability is straightforward.

Proposition 5: For any $x \in X$ and Borel set $A \in \mathcal{B}(X)$, we have

$$
P_{x}\left[\operatorname{Reach}_{\infty}(A)\right]=B_{A} 1(x)=P_{A} 1(x)=P_{x}\left[T_{A}<\infty\right]
$$

The excessive function $B_{A} 1$ is called sometimes the equilibrium potential of the set $A$. From the equality (10) that, in the computation of the reach set probabilities, we are not interested on those trajectories of the process $\left(x_{t}\right)$ which start in $A$. These probabilities are trivially equal to 1 .

\section{B. Optimal Stopping Problem}

In Markov process theory, the existence of the réduite of a bounded measurable function $g: X \rightarrow \mathbb{R}$ (with respect to the resolvent $\mathcal{V}$ ) is proved using different ideas from the ones used in potential theory. In fact, this existence is based on the following equality [16]:

$$
R g(x)=\sup \left\{E_{x}\left[g\left(x_{S}\right) 1_{\{S<\infty\}}\right] ; S \text { stopping time }\right\} \text {. }
$$

It is easy to see that right hand side of the equality (11) is related with the so-called optimal stopping problem (OSP) associated with a Markov process.

For a particular class of SHS, namely for piecewise deterministic Markov processes (PDMP), the OSP has been studied by M.H.A. Davis in [10]. We briefly recall the OSP definition for a strong Markov process $M=\left(\Omega, \mathcal{F}, \mathcal{F}_{t}, x_{t}, P_{x}\right)$ taking values in a Lusin space.

Let $\Sigma$ denote the set of stopping times (finite or not) with respect to the filtration $\left\{\mathcal{F}_{t}\right\}$ (i.e. $\tau \in \Sigma \Leftrightarrow \forall t,\{\tau \leq t\} \in$ $\mathcal{F}_{t}$ ). Consider $g: X \rightarrow \mathbb{R}$ a bounded measurable function called the reward function (the interpretation being that if we stop the process at a point $x \in X$ we obtain a reward $g(x)$ ). Obviously, the definition of OSP requires some integrability conditions over the paths of $M$ (see, for example [16], for more details). Let $\left(y_{t}\right)_{t \geq 0}$ be the reward process defined by $y_{t}=g\left(x_{t}\right), t \geq 0$.

The maximal payoff function (or the value function, in the terminology of [10]) is

$$
v(x):=\sup \left\{E_{x} y_{\tau} \mid \tau \in \Sigma\right\} .
$$

The value function has been characterised in terms of the minimal excessive function lying above the reward function [16]. In the light of the definitions presented in the previous subsection, this means that the reduite of $g$ coincides with the value function (12). Moreover, the value function can be characterized by means of the smallest supermartingale (called Snell's enevelope) dominating the reward process.

\section{Stochastic reachability as an optimal stoping problem}

Now, let us consider a target set $A \in \mathcal{B}(X)$ in the context of the reachability problem. It is easy to observe that if we set the reward function $g$ to be equal with the indicator function of $A$, i.e. $g:=1_{A}$, according to the characterization of the reach set probability derived in the previous subsection we obtain the following result:

Proposition 6: If $A \in \mathcal{B}(X)$ then the reach set probability $P .\left[\operatorname{Reach}_{\infty}(A)\right]$ is the value function of the reward process $y_{t}=1_{A}\left(x_{t}\right)$, i.e.

$$
P_{x}\left[\operatorname{Reach}_{\infty}(A)\right]=\sup \left\{E_{x} 1_{A}\left(x_{\tau}\right) \mid \tau \in \Sigma\right\}, \forall x \in X .
$$

Considering also the potential theory perspective on the stochastic reachability problem, we can also derive a characterization of $P_{x}\left[\operatorname{Reach}_{\infty}(A)\right]$ in terms of a functional inequality involving the quadratic form $\mathcal{E}$ associated to the generator $\mathcal{L}$ (see [7]) as follows:

Proposition 7: If $A \in \mathcal{B}(X)$ then the reach set probability $P$. $\left[\operatorname{Reach}_{\infty}(A)\right]$ (or the equilibrium potential $A$ ) is the unique excessive function $u$ satisfying: (i) $\mathcal{E}(u, v)=$ $(-\mathcal{L} u, v) \geq 0, \forall v \in D[\mathcal{E}]$ (domain of $\mathcal{E}), v \geq 0$ on $A$; (ii) $u=1$ on $A$.

\section{Computation}

For PDMP, the OSP has been successfully solved in [10], but for bounded continuous reward functions. More, in [10], it is provided a computational method to estimate the value function. This value function is also characterized in terms 
of a functional equation involving the infinitesimal operator of a PDMP. For diffusions with jumps (which represent also a particular class of SHS) some continuity properties and upper bounds have been investigated in [16].

For $A \in \mathcal{B}(X)$, let us introduce the reachability function $w: X \rightarrow[0,1]$ defined as

$$
w_{A}(x):=P_{x}\left[\operatorname{Reach}_{\infty}(A)\right] .
$$

The indicator function $1_{A}$ is a measurable bounded function, but it is not continuous, so the associated value function defined by (12), which, in this case, coincides with $w_{A}$, may fail to be continuous. Then the computational methods via dynamic programming, summarized in [1], might not work in our case.

But for the case when $A$ is an open/closed set, we can characterise $w$ as the viscosity solution of the variational inequalities associated to the infinitesimal operator $\mathcal{L}$ of the process (see the next paragraph), since the value function (which is, in this case, equal with the indicator $1_{A}$ ) is a semicontinuous function (lower/ upper). Results on the existence and regularities of the viscosity solution ${ }^{5}$ for general Markov processes when the value function is semicontinuous can be found in [9].

Theorem 8: If $A \subset X$ is an open set such that the reachability function $w_{A}$ defined by (13) satisfies

$$
w_{A}(x)=1 \text {, for all } x \in \partial A
$$

where $\partial A$ is the topological border of $A$, then $w_{A}$ is a viscosity solution of

$$
\min \left\{-\mathcal{L} w_{A}, w_{A}-1_{A}\right\}=0
$$

where $\mathcal{L}$ is given by (6).

Proof: The result is a consequence of the theorem on viscosity solution from [9], if the hypotheses of that theorem are fulfilled. We have to check that: (a) the martingale problem for $\mathcal{L}$ is well-posed (which is true, see [8]), (b) the operator semigroup has the Feller property (this is also true according to [22], since the generator is a pseudo-differential operator), and (c) the following inequality holds

$$
w_{A} \geq\left(1_{A}\right)^{*},
$$

where $\left(1_{A}\right)^{*}$ is the upper semicontinuous envelope ${ }^{6}$ of $1_{A}$. Clearly, $\left(1_{A}\right)^{*}=1_{\bar{A}}$, where $\bar{A}$ is the closure of $A$. The inequality (15) results from (10) and (14).

Since the hypotheses from [9] are satisfied, the conclusion of the theorem is true.

\section{CONCLUSIONS}

In this paper, we have investigated different new characterizations of the stochastic reachability problem for SHS. These are expressed in terms of supermartingales, mean hitting times, reduite functions, hitting operators and optimal

\footnotetext{
${ }^{5}$ Here, the notion of viscosity solution is understood in a general sense, see, e.g. [9].

${ }^{6}$ The upper semicontinuous envelope is defined as in the viscosity literature.
}

stopping problems. They are the result of the examination of this problem from different angles corresponding to the mathematical theories that can be utilized in the study of the stochastic processes, which appear in the semantics of SHS.

Finally, these "new insights" on stochastic reachability have conducted to the result, which says the reach set probabilities can be computed as solutions of some equations with respect to the infinitesimal operator. This opens the direction to apply dynamic programming and/or linear programming to compute numerically these probabilities.

\section{REFERENCES}

[1] Bingham, N.H., Peskir, G.: Optimal Stopping and Dynamic Programming. Lecture Notes 1 (2006), Probab. Statist. Group Manchester.

[2] Blom, H.A.P.: Stochastic Hybrid Processes with Hybrid Jumps. Proc. IFAC Conference ADHS, (2003): 361-365.

[3] Blom, H.A.P., Lygeros, J. (Eds.): "Stochastic Hybrid Systems: Theory and Safety Critical Applications". LNCIS 337 (2006).

[4] Bujorianu, M.L., Lygeros, J.: Towards Modelling of General Stochastic Hybrid Systems. In [3]: 3-30.

[5] Bujorianu, M.L., Lygeros, J.: Reachability Questions in Piecewise Deterministic Markov Processes. Hybrid Systems: Computation and Control, 6th International Workshop, LNCS 2623 (2003): 126-140.

[6] Bujorianu, M.L . Extended Stochastic Hybrid Systems and their Reachability Problem. Hybrid Systems: Computation and Control 7th International Workshop, LNCS 2993 (2004): 234-249.

[7] Bujorianu, M.L., Blom, H.A.P., Hermanns, H.: Functional Abstractions of Stochastic Hybrid System. In Proc. of IFAC ADHS, (2006): 160-165.

[8] Bujorianu, M.L., Lygeros, J.: General Stochastic Hybrid Systems: Modelling and Optimal Control. Proc. 43th CDC, IEEE Press (2004): 182-187.

[9] Ceci, C.: Regularity of the value function and viscosity solutions in optimal stopping problems for general Markov processes. Stoch. Stoch. Rep. 74(3-4) (2002): 633-649.

[10] Davis, M.H.A.: "Markov Models and Optimization", London, (1993).

[11] Dellacherie, C., Maisonneuve, B., Meyer, P.-A.: "Probabilités et Potentiel. Ch. XVII à XXIV. Processus de Markov (fin). Complements de calcul stochastique". Hermann, Paris (1992).

[12] Doob, J.L.: "Classical Potential Theory and Its Probabilistic Counterpart". Berlin (1984).

[13] Doob, J.L.: "Stochastic Processes". Wiley, New York (1953).

[14] Dynkin, E.B.: The optimum choice of the instant for stopping a Markov process. Soviet. Math. Dokl. 4 (1963): 627-629.

[15] Dynkin, E.B.: "Markov processes". Moscow (1963), Vol. II of English translation. Springer (1965).

[16] El Karoui, N., Lepeltier, J.-P.; Millet, A.: A Probabilistic Approach to the Reduite in Optimal Stopping. Probab. Math. Statist. 13 (1992), no.1, 97-121.

[17] Ethier, S.N., Kurtz, T.G.: "Markov Processes: Characterization and Convergence". New York: John Wiley and Sons, (1986).

[18] Hespanha, J.P., Singh, A.: Stochastic Models for Chemically Reacting Systems using Polynomial Stochastic Hybrid Systems. Int. J. on Robust Control Special Issue on Control at Small Scales: Issue 1, 15(15) (2005): 669-689.

[19] Hespanha, J.P.: Stochastic Hybrid Systems: Application to Communication Network. Hybrid Systems: Computation and Control 7th International Workshop, LNCS 2993 (2004): 387-401.

[20] Hu, J., Lygeros, J., Sastry, S.: Towards a Theory of Stochastic Hybrid Systems. In N. Lynch and B. Krogh (Eds.), Proc. of Hybrid Systems: Computation and Control, LNCS 1790 (2000): 160-173.

[21] Hunt, G.A.: Markov Processes and Potentials I, II, III. Illinois J. Math. 1: 1 (1957): 44-93; 1: 3 (1957): 316-369; 2: 2 (1958): 151-213.

[22] Kolokoltsov, V.N.: On Markov Processes with Decomposable PseudoDifferential Generators. Stoch. and Stoch. Rep. 76(1) (2004): 1-44.

[23] Pola, G., Bujorianu, M.L., Lygeros, J., Di Benedetto, M. D.: Stochastic Hybrid Models: An Overview with applications to Air Traffic Management. Proc. ADHS, (2003): 45-50.

[24] Ville, J.: "Etude Critique de la Notion de Collectif". Gauthier-Villars, Paris (1939). 